\title{
Basic Principles of the Activity and Synergetic Approach as a Means of Interdisciplinary Research
}

\author{
L.M. Bolsunovskaya ${ }^{1, a}$, I.A. Kuprieva ${ }^{2}$, and S.I. Gamzatova ${ }^{3}$ \\ ${ }^{1}$ Tomsk Polytechnic University, 634050 Lenin Avenue, 30, Tomsk, Russia \\ ${ }^{2}$ Belgorod State National Research University, 308001 Pobedy st., 85, Belgorod, Russia \\ ${ }^{3}$ Tomsk Polytechnic University, 634050 Lenin Avenue, 30, Tomsk, Russia
}

\begin{abstract}
The paper considers the problem of interdisciplinarity as the main tendency of today's science to unite the methodology and terminology and adapt them to any academic field in order to achieve more objective results in the study of complex phenomena. This article is a descriptive one and it deals with problems of terms' interpretations and their adaptation to the anthropocentric research viewed by scientists of different academic schools. The main idea of the article is to find the most relevant and systemic definition of the given terms, which could be used as a tool in further linguistic analysis applied to the coming research of the authors. Special attention is paid to the description of the Activity Theory from different aspects of its interpretation: psychological, sociological, linguistic; to the explanation of the correlation between the epistemic structure of knowledge and an open non-linear synergetic bilateral (conceptual and lexical-semantic) system influenced and motivated by processual factors to evolution in the modern discourse as the communicative activity of the interpreter and the constructor of discourse.
\end{abstract}

\section{Introduction}

Modern scientific world tends to globalization and integration. It has entered the post non-classical stage of development with its new rational humanitarian anthropomorphism and high standards and requirements to research methodology. Thus, science as it is, does not simply represent any separate field any more. It develops universal methods that can be applied to any research in order to optimize it and create universal explanatory principles of social reality. One of such principles is called the Activity Approach Principle, which is essentially focused on the implementation of an explanatory principle for the various processes and phenomena arising in society as it is primarily explained in sociology and philosophy. But in order to enable it to fulfil its explanatory function in a large-scale scientific study, the researcher needs to fill it with the necessary content, specify it, consequently, classify and combine with more or less traditional methodology principles. To do this, we should take into consideration that the basic element of this theory is activity itself and it stands for a certain type of human existence in the world and his or her interaction in society. Originally this term was introduced in psychology, sociology and philosophy to describe the anthropocentric factor and its role in the evolution of society. And such tendency to study any psychological or sociological phenomena from the point of view of the activity approach remained stable for a long time. But at the same time, scientists were also interested in the language representation of the activity theory, postulated correlation of thinking and language in the cognitive activity of the person as well. In other words the notion or some concept of any phenomenon of the outer world stored in the individual's mind is not its simple reflection but the result of a subjective refraction through the prism of abstraction, generalization and imagination. This reflective feature of the meaning, characterized by an activity or procedural (in the terms of cognitive science) approach transforms experience and transmits it by lexical semantics. The first to define a lexical meaning as the concentration of the experience of society, its practical activity including the results of such activity and the process of activity as such was the famous Russian psychologist, the founder of the activity approach to semantics, L.S. Vygotsky. His point of view was later supported by G.P. Schedrovitsky who described a lexical meaning as the systemic activity of humanity towards material signs and its verbal reflection [1]. Covering the method of work and way of thinking, the author refers to the identification and interpretation of the basic principles of activity, which primarily include the idea of activity as a reflection of mental operations (including the categorization of operations),

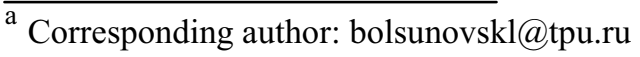


aiming at the process of understanding, searching and identification of patterns of functioning of extralinguistic data in our consciousness. It allows G.P. Schedrovitsky to state the prior position of activity and the secondary position of the knowledge and continuous principle of knowledge backlog [1]. Taking into account the priority of activity scientists deduced the third principle - a system that reflects the situation that the categories themselves - both ontological and methodological foundations - are the result of activities and subsequently affect them. This principle, according to G.P. Shchedrovitsky, has the mechanism of a closed circle. The researchers mainly noted the importance of social, external and internal psychological factors in the formation of lexical semantics [2;3]. The meaning is identified with the process and opposed to things, on the one hand, and is associated with the hierarchy of dynamic processes rather than with the system of objects, on the other. This explains the existence of the basic synonymous term procession and allows naming this approach as the basic one for explaining non linguistic and linguistic facts. Further in this article we will take into consideration the basis of this theory, adapt it and combine with other instruments of scientific research.

\section{Materials and methods}

This article is a descriptive one. Its main goal is not only to characterise and describe the use of the Activity and Synergetic Approach in combination with modern research methods, but to show the basic principles of its application while explaining the mechanisms of synergetic evolution. The ongoing research is based on the systemic approach to the term interpretation, analysis of different points of view. We also take into consideration the interdisciplinary approach of modern science, which allows us to search objective reasons of our choice.

\section{Results and discussion}

Taking into consideration the basic principles of the activity approach, we assume that the situation itself, the conditions of the outer world influence the individual and this motivates his or her proper verbal reaction, that is the choice of the language means (lexical, syntactical with a pragmatic component) expressing the thought. This allows supposing that not only the choice of a lexeme but the modification of its semantics has an anthropocentric nature. In other words it is predetermined by the result of the joint activity of the participants of the communication act. This exactly means the priority of speech activity of communicants over selection of linguistic signs and constructing statements. Moreover taking into consideration psychological, sociological data, we suppose that formation of semantics is viewed as a dynamic process, concerning mainly the level of mental processes and taking into account their instrumental role, so the term activity can be substituted by the term process.
As it is mentioned by the founders of the Activity Approach the key factors shaping and modifying semantics are the structure of activity, the nature of the communicative act, the context, the potential meaning of expressions, speech strategies and search tactics, taking into consideration the units' nomination. Moreover, the chosen methodology of the Activity Approach allows revealing and explaining the principle of inclusion and the influence of social factors on language environment, the semantics of signs, as a semantic unit of language, consciousness while constructing conceptual models of the world.

According to the basic principles of cognitive science the knowledge of the outer world is reflected in person's consciousness and is organized as a hierarchical structure or a formalized model of information in consciousness. This structure has a more or less invariant basis which serves to its identification and variable elements that can be modified due to various factors in the speech activity. This data stored in the consciousness can be verbalized by language means, which transmit social experience due to the actualization of invariant information and some individual knowledge to variable ones. The invariant basis is transmitted from one generation to the other in the course of the development of society. The latter depends on the person's activity including individual actions, which are coordinated by its specific objectives, conditions and modes of action (or operations). In other words the basic principles of word semantics' organization contain a more or less generalized detailed image of the object, but also person's attitude to the subject. This attitude forms a correlation between meanings of both communicants [4]. In other words the epistemic structures of consciousness meet the requirements of modifications under social conditions and communicative situation as an activity process. The existence of dual interpretation of knowledge (the basic knowledge transmitted in the lingvo culture, on the one hand, and subjective attitude and conceptualized experience, on the other) allows supposing that the invariant basis of the mental structure serves to its identification and the variative part serves to its modification and receiving the incoming knowledge flow from the outer world. The latter determines understanding between communicants. In order to show the process of understanding and transmitting the sense we distinguish the following roles of the communicants: an interpreter (the addressee) and a constructor (addressant).

As it has already been mentioned, the very invariant part of the structure does not modify under subjective circumstances. It can be named the Level of the Highest Abstraction. The affective and emotional background is stored on the Level of the Lowest Abstraction or the level of the concrete communicant. Additional information about personal experience and personal associations is stored particularly here. This level is being constantly modified in accordance with the goals and conditions of individual's activities in communication and socialisation. These two layers existing in coherence provide the trinity of denotative, connotative and pragmatic components. The scheme of the two-layer 
mental organization can be represented in the following way:

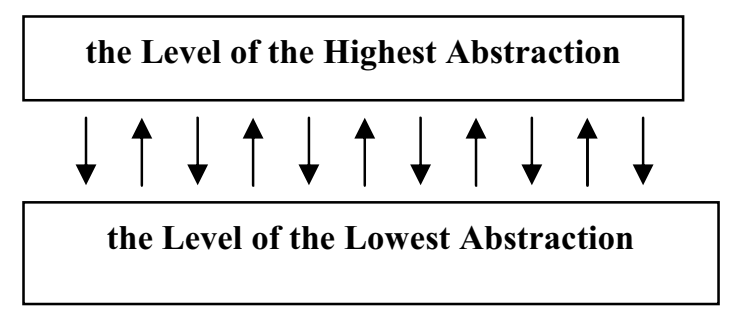

Fig. 1. The Two-Layer Mental Organization Scheme

The Level of the Lowest Abstraction, as it was mentioned earlier, is flexible to change as it contains peripheral knowledge constantly enlarged and enriched. It can be schematically represented as the following:

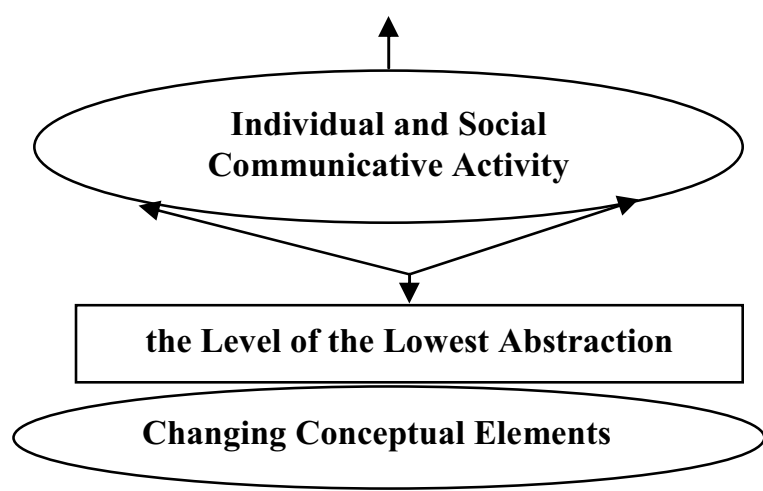

Fig. 2. Organization of the Level of the Lowest Abstraction

Being open to any change in the constant process of incoming knowledge flow from the outer world in the periods of epistemic mental structures' interaction at the Level of the Lowest Abstraction, presumably, the mental structure is related to the synergetic system. This supposition of the authors comes from that fact that it is open and non-linear. Its constant interaction with other mental structures provides a constant flow of resources from the outer world, and this new incoming information needs to meet the requirements of the system in order to be identified in accordance with the parameters of order. The new flow of information adapts to the system parameters in the process of evolution when the system spends its own recourses and new recourses in order to modify in the long run. This evolution occurs at the conceptual level, but according to cognitive linguistics the data stored in a mental structure is verbalized by lexemes. And this fact also allows speaking of the existence of a whole lexical-semantic system of mental structure's verbalizers. Thus, the described system of the epistemic structure may be called bilateral. In this case it is possible to identify the principles of the latter in its natural state of representation, or so to say, identify the lexical parameters of the system. The order parameters, the equilibrium state of the system in this case is more objectively seen at the contextual level, i.e. while they are verbalized in the discourse.

In other words, the equilibrium state of the bilateral system existing as a conceptual and as a lexical-semantic one is vividly seen in isolation, while it is not inverted in the communication process (communicative activity) and its conceptual components as well as lexemes are stored in the mind of the communicant. As soon as the process of communication starts, for example, there arises a necessity of the use of lexemes non typical of their semantics, in occasional meaning so to say, the bilateral system starts its adjustment procedure at the conceptual level which changes the semantics of the lexemes within the acceptable minimum of the system adjustment. The latter is the result of intra-systemic (occurring within the concept) modification processes. They are often fixed in the dictionaries and other lexicographical sources and therefore they are no longer associated with neologisms by the communicants. The meaning mentioned above is the result of extra-systemic modifications when the whole system of concept verbalizers interacts with other conceptual systems and receives new information from the outer world. The results of such processes are idiomatic expressions, connotations [5].

The change of meaning occurs in the activity process in discourse. Having analyzed the definitions of the discourse given by scientists, we can conclude that the text is a static unity, of formal design, which comes alive in the discursive space in real-time due to non-linguistic factors. Accordingly, with respect to the ongoing research discourse will be treated as a living dynamic complex including several text units of different kinds, usually unrelated with each other. However, such a broad definition of discourse including the specification of its components is very important to us because the vast abstract notion like discourse should be considered on the basis of an analysis of its elements and the relations between them.

Works by W. Chafe also seem relevant mainly in terms of the wording of cognitive-pragmatic criteria. The fact is that the scientist was always interested in the sphere of consciousness and language in their interaction and correlation [6]. In this regard, the attention of the linguist is focused on such problems as language basis of mechanisms based on the study of the processes of consciousness and problems of written/spoken language in terms of their comparison. In the structural aspect W. Chafe defines discourse as an association of intonation units, i.e. the so-called quants of discourse, each of which is equal to one focus of consciousness or a new item in terms of content. Available information (or intermediate stage) between old and new is called a topic. Within the information conversion that includes intonation units, the ideas of objects, events and qualities, understood as concepts. Such concepts, according to W. Chafe, may exist in three stages of activation: active state, identical to the classical name of this information; semi-active state, or information that is available, it occupies an intermediate position between the old and new and inactive, correlated with new information.

If we assume that the communicative activity is profiled in discourse, we can notice a phased activation of the latter as a result of the progressive discourse interpreter's consciousness focus drift. Description of 
mechanisms of mental structures deployment and modification under processual (activity) factors in modern discourse in this study is based on two key principles: 1) taking into account the interpreter's perception of the meaning in the discursive space in the process of information decoding; 2) taking into account the constructor's intention in the process of creating a discourse while coding the information. It seems that such a many-sided research of sense transition in the context of discourse in process of mental structures' actualization, their deployment, will contribute to a more objective study. Accordingly, such structural units exist in different states of activation in the mind of discourse interpreter. Taking into consideration that fact that basic lexemes play a catalytic role for the discourse interpreter's consciousness in the process of mental structures' deployment we will research this problem later. Note, however, that such an order at a particular stage does not exclude the step when consciousness correlates conceptual information derived from the discourse with a proposition. Macrostructures are eventually represented by propositions of the sentence and its content, which is correlated with the mapping. These macropropositions are encoded in text frames, text and topic coherence. The functions of text structure planning and processing are given to communicants. Thus, the discourse can be studied in the text, and in the cognitive dimensions, which in principle would allow to consider it more objectively and systematically taking into account the maximum of non-linguistic factors, including the social situation. Extralinguistic factors such as, for example, conversation implicature, serve as a kind of an additional message to the recipient and include the usual linguistic data, contextual information, as well as give an idea of the general principle of cooperation or coherence. The principle of cooperation precisely reflects the essence and the connection of information contained in the discourse, which allows perceiving it as a single integrated dynamic and harmonious unity.

\section{Conclusion}

1. In the present study, we rely on the data given by psychology, sociology, philosophy associated with the Activity Approach, activity is a fundamental principle of knowledge, it contributes to the development of the primary forms of thinking, it can be considered as awareness of the person's own life or experience of mankind.

2. The knowledge is structured and stored in a certain way in the human mind as a certain formal structure is dynamic and can change depending on the activity conditions.

3. Knowledge and experience obtained due to individual's and social activities is stored and modified in the form of certain mental constructs, which contain two different storage levels.

4. The storage of information in the mind is structured vertically: the base invariant level (the Level of the Highest Abstraction) includes highly abstract information, which is broadcast to succeeding generations. The level of the mentality of a particular individual (the Level of the Lowest Abstraction), in addition to basic information, stores the knowledge and associations, emotional and affective components that are typical of individual consciousness.

5. The level of the mentality of a particular individual is subjected to constant metamorphoses and changes according to his or her experience.

6. Knowledge transfer is carried out through the lexical units that are organized around a mental structure as the attractor.

7. Being represented at the conceptual and language level, the mental structure can be named a bilateral system which is a non-linear, open, non-equilibrium, dissipative synergistic system.

8. Violation of the relative nonequilibrium system order is a result of the choice of occasional lexemes in nomination in the active communication process.

9. The main factors triggering fluctuations in the bilateral system are the key factors of procedural approach (Actovoty Approach).

10. Accordingly, to determine the mechanism of verbalization of mental structures we proceed from the assumption that the lexical-semantic synergetic system comes into a state of chaos due to procedural factors acting at the level of mentality particular communicant in terms of discourse.

11. According to the author's participation in the interpretation of the meaning in discourse, a triad of denotative, connotative and pragmatic components is quiet obvious.

In summary, we believe that the level of variability of a synergetic system depends on its ability to adjust itself under the influence of processual factors in the construction and interpretation of discourse.

\section{References}

1. G. P. Shhedrovickij, Znak i dejatel'nost' (M. Vost. Lit., 2005)

2. E. V. Gelderen, The Linguistic Cycle. Language Change And The Language Faculty (Oxford University Press, 2011)

3. A. Wray, Formulaic Language: Pushing the Boundaries (Oxford University Press, 2008)

4. G. Ja. Solganik, Stilistika sovremennogo russkogo jazyka i kul'tura rechi (M. Academia, 2005)

5. Traugott, Elisabeth \& Dasher. Regularity in Semantic Change (Cambridge: Cambridge University Press, 2002)

6. W. Chafe, Discourse, Consciousness, and Time: The Flow and Displacement of Conscious Experience in Speaking and Writing (University of Chicago Press, 1994) 